\title{
Free and forced vibrations of shell structures interacting with liquid
}

\author{
V. Gnitko, V. Naumenko, U. Ogorodnik \& E. Strelnikova \\ Institute for Mechanical Engineering Problems of the Ukrainian \\ Academy of Sciences, Ukraine
}

\begin{abstract}
The method for dynamic analysis of shell structures partially filled with liquid is presented in this paper. The method relies on determining the fluid pressure from the system of singular integral equations. The axisymmetric compound shell of revolution is chosen as the model of capacity for liquid storage. The shell is considered to be a thin one, and Kirghoff-Lave linear theory hypotheses are applied. The liquid is ideal and incompressible. The coupled problem is solved using a combination of reduced BEM and FEM. Differential equations of the transient problem are solved numerically by the Runge-Kutta method of 4th and 5 th order. Numerical simulation of free and forced vibrations of the elastic and rigid shells filled with the incompressible fluid under different loadings has been carried out.
\end{abstract}

Keywords: fluid-structure interaction, free and forced vibrations, boundary and finite element methods.

\section{Introduction}

Different engineering areas such as aerospace and chemical industry, power machine building, wind power engineering and transport extensively use thinwall structural elements operated under excess process loads. Usually they are also filled with oil, flammable or toxic liquids. Such facilities are fuel tanks, liquid storage tanks, oil and propellant storage containers. Destruction of these tanks by seismic action or shockwaves from a nearby explosion can lead to environmental catastrophe. Complex experimental investigation of loading processes is difficult and sometimes impossible for various reasons. Hence mathematical modeling of physical processes with the help of advanced 
computer engineering is a basic approach for these problems. Currently it has also been recognized that several containers and storages with toxic or flammable substances are located in areas formerly considered as seismically safe, and therefore they have been designed ignoring possible horizontal loads according to obsolete seismic standards. The information from Donetsk [1] specifies: strata shearing will occur not only as the result of earthquakes and explosions, but also due to soil falls in coal mines. There are a lot of coal mines in Donbass that are not operated since 1920s. Maps of these mines were lost during revolutions and wars. Note that in Donbass there is a lot of oil storages located near such mines. The impacts due to soil falls can be also modeled as horizontal seismic loads. It should be noted that not only estimation of operating equipment, but also reliability-focused design of new high-performance machines and structures requires defining the strength characteristics of their elements. These data allow evaluating the ultimate strength of a structure under shock or seismic action, isolate spurious resonance frequencies, and identify the most hazardous zones at the design stage. A distinguishing feature of these structures is that they operate interacting with the air or water environment. Owing to this, to define the strength characteristics it is necessary to solve problems of hydro-elasticity, i.e. to find stress and strain fields and vibration frequencies considering fluid or gas pressure forces acting on the elastic body. Numerical methods are especially useful when the geometry of container is complicated and the sloshing in the container cannot be analytically investigated. Various approaches have been proposed to research fluid-structure interaction, including the finite difference methods [2], the finite element methods [3], the boundary element methods [4-8]. The research findings are summarized in [9].

In this paper the coupled problem of free and forced vibrations of shell structures interacting with the fluid is under consideration. For its solution we use combination of reduced finite and boundary element methods. The analysis consists of several stages, each represents a separate task. The frequencies and modes of shell vibrations in a vacuum are defined by the first stage. Displacement vector, that is the solution of the hydrodynamic problem, is sought as a linear combination of the natural modes of shell vibrations in vacuum. So at the second stage we define the frequencies and free vibrations modes of elastic shell without including the force of gravity. Thirdly, we obtain the frequencies and free vibrations modes of liquid in rigid shell under force of gravity. The latter two problems are solved using reduced BEM. Then we come to second order system of differential equations for forced vibrations of the shell partially filled with liquid and solve it numerically using Runge-Kutta method.

\section{Problem statement}

Let us consider the coupled problem for shells interacting with the liquid. In this study the contained liquid is assumed to be inviscid and incompressible. 
Governing equations of motion for the liquid-structure system subjected to disturbing force are given by

$$
\left\{\begin{array}{c}
L_{11} u+L_{12} v+L_{13} w=\rho h \frac{\partial^{2} u}{\partial t^{2}}+Q_{1}, \\
L_{21} u+L_{22} v+L_{23} w=\rho h \frac{\partial^{2} v}{\partial t^{2}}+Q_{2}, \\
L_{31} u+L_{32} v+L_{33} w=\rho h \frac{\partial^{2} w}{\partial t^{2}}+P_{l}+Q_{3} .
\end{array}\right.
$$

Here $u, v, w$ are the displacement components; $L_{i j}^{(k)}(i, j=1,2,3)$ are differential operators; $Q=\left(Q_{1}, Q_{2}, Q_{3}\right)$ is the vector of disturbing force; $\rho$ is the material density; $h$ is the shell thickness, $P_{l}$ is hydrodynamic pressure.

Let $v_{x}, v_{y}, v_{z}$ be the components of fluid velocity, then the incompressibility condition can be written as follows:

$$
\operatorname{div} \vec{v}=\frac{\partial v_{x}}{\partial x}+\frac{\partial v_{y}}{\partial y}+\frac{\partial v_{z}}{\partial z}=0
$$

Suppose that disturbing force $Q$ is the sum of vertical gravitational and seismic horizontal forces. Then the fluid displacement vector can be expressed in the form

$$
-\rho_{l} \frac{\partial v_{x}}{\partial t}=\frac{\partial p}{\partial x}+\rho_{l} a_{S}(t) ; \quad-\rho_{l} \frac{\partial v_{y}}{\partial t}=\frac{\partial p}{\partial y} ; \quad-\rho_{l} \frac{\partial v_{z}}{\partial t}=\frac{\partial p}{\partial z}+\rho_{l} g .
$$

Here $\rho_{l}$ is the liquid density, $a_{S}(t)$ and $g$ are seismic and gravitational accelerations. So the liquid pressure could be expressed as the sum of dynamical and static pressures

$$
P_{l}=p+\rho_{l} g z+\rho_{l} a_{s}(t) x .
$$

Suppose that flow is the potential

$$
V_{x}=\frac{\partial \phi}{\partial x}, \quad V_{y}=\frac{\partial \phi}{\partial y}, \quad V_{z}=\frac{\partial \phi}{\partial z} .
$$

Due to (2) the velocity potential $\phi$ satisfies the Laplace equation

$$
\frac{\partial^{2} \phi}{\partial x^{2}}+\frac{\partial^{2} \phi}{\partial y^{2}}+\frac{\partial^{2} \phi}{\partial z^{2}}=0 .
$$

The hydrodynamic pressure, according to the Cauchy-Lagrange integral, can be represented as follows

$$
\frac{p}{\rho_{l}}=-\frac{\partial \phi}{\partial t}-g z+\frac{p_{0}}{\rho_{l}}-a_{s}(t) x
$$

where $z$ is vertical coordinate of a point in liquid. 
We denote a moistened surface of a shell through $\mathrm{S}_{1}$ and a free surface as $\mathrm{S}_{0}$. Let refer the Cartesian coordinate system 0xyz connected with a shell (Figure 1). The free surface of the liquid $\mathrm{S}_{0}$ coincides with the plane $\mathrm{x} 0 \mathrm{y}$ in unperturbed state. Let liquid storage tank is subjected to a dynamic load. To obtain the boundary equations on the free surface we have formulated dynamic and kinematics boundary conditions. The dynamic boundary condition consists in equality of the liquid pressure on the free surface to atmospheric one. The kinematics boundary condition requires that liquid particles of the free surface remain on it for all subsequent motion.
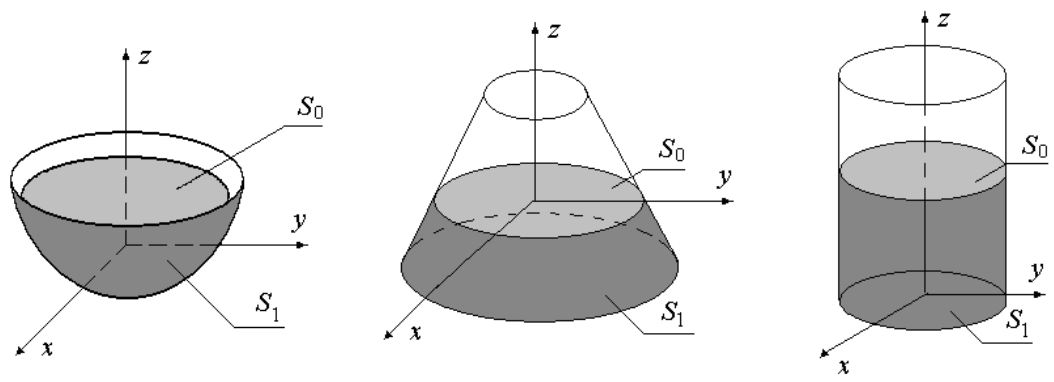

Figure 1: Shells of revolution partially filled with liquid.

So we obtain the following boundary value problem for defining the unknown functions $\mathbf{U}$ and $\phi$.

$$
\begin{gathered}
\boldsymbol{L} \boldsymbol{U}+\boldsymbol{M} \ddot{\boldsymbol{U}}+\rho_{l} \dot{\phi}+g z=Q ; \\
\frac{\partial \phi}{\partial n}=\frac{\partial w}{\partial t}, P \in S_{1} ; \frac{\partial \phi}{\partial n}=\dot{\zeta}, P \in S_{0} ; \dot{\phi}+g \zeta=0, P \in S_{0}
\end{gathered}
$$

where $\mathbf{L}, \mathbf{M}$ are matrix operators corresponding to $L_{i j}^{(k)}$ and inertial components. We will seek the natural modes of shell vibration in the fluid as follows

$$
U(x, y, z, t)=\sum_{k=1}^{m} c_{k}(t) u_{k}(x, y, z)
$$

where functions $u_{k}(x, y, z)$ are modes of natural vibrations in vacuum, $c_{k}(t)$ are unknown factors.

We will seek function $\phi$ as a sum of two potentials $\phi=\phi_{1}+\phi_{2}$. Potential $\phi_{1}$ corresponds to the problem of vibrations of elastic shell with weightless fluid $(g=0)$. To determine $\phi_{1}$ we obtain the following boundary value problem:

$$
\nabla^{2} \phi_{1}=0, \frac{\partial \phi_{1}}{\partial n}=\frac{\partial w}{\partial t}, P \in S_{1}, \frac{\partial \phi_{1}}{\partial t}=0, P \in S_{0} .
$$


Here $w(x, y, z, t)=\sum_{k=1}^{m} w_{k}(x, y, z) c_{k}(t)$ and functions $w_{k}(x, y, z)$ are normal modes of natural vibrations of the shell in vacuum. From equations (3) and second one from (6) it follows that

$$
\phi_{1}(x, y, z, t)=\sum_{k=1}^{m} \phi_{1 k}(x, y, z) \dot{c}_{k}(t) .
$$

To determine $\phi_{1 \mathrm{k}}$ we have the next set of boundary value problems:

$$
\nabla^{2} \phi_{1 k}=0, \frac{\partial \phi_{1 k}}{\partial n}=w_{k}, P \in S_{1}, \phi_{1 k}=0, P \in S_{0} .
$$

To determine $\phi_{2}$ we have to solve the problem of fluid vibrations in rigid vessel including gravitational force

$$
\nabla^{2} \phi_{2}=0, \frac{\partial \phi_{2}}{\partial n}=0, P \in S_{1}, \frac{\partial \phi_{2}}{\partial n}=\dot{\zeta}, P \in S_{0}, \dot{\phi}_{2}+g \zeta=0, P \in S_{0} .
$$

The last equation in (9) follows from equation (3) and represents dynamic condition on the free surface. Differentiating this equation with respect to $t$ we come to the following equation for velocity potential in the rigid vessel:

$$
\ddot{\phi}_{2}+g \frac{\partial \phi_{2}}{\partial n}=0, P \in S_{0} .
$$

Let us seek for the solution of this problem in the next form:

$$
\phi_{2}(x, y, z, t)=e^{i \kappa t} \psi(x, y, z) .
$$

For function $\psi$ we have the following problem of free harmonic fluid vibrations:

$$
\nabla^{2} \psi=0, \frac{\partial \psi}{\partial n}=0, P \in S_{1}, \frac{\partial \psi}{\partial n}=\frac{\kappa^{2}}{g} \psi, P \in S_{0} .
$$

Solving this problem one can obtain the number of eigenvalues $\kappa_{\mathrm{k}}$ and corresponding eigenfunctions $\phi_{2 \mathrm{k}}$. After equation (11) is solved we are looking for function $\phi_{2}$ in the form

$$
\phi_{2}(x, y, z, t)=\sum_{k=1}^{n} \dot{d}_{k}(t) \phi_{2 k}(x, y, z) ; \phi_{2 k}(x, y, z)=\psi_{k}(x, y, z) .
$$

So we have $\phi=\phi_{1}+\phi_{2}$, where

$$
\phi_{1}(x, y, z, t)=\sum_{k=1}^{m} \phi_{1 k}(x, y, z) \dot{c}_{k}(t), \phi_{2}(x, y, z, t)=\sum_{k=1}^{n} \dot{d}_{k}(t) \phi_{2 k}(x, y, z)
$$

Then

On free surface it is required

$$
\nabla^{2} \phi=\nabla^{2} \phi_{1}+\nabla^{2} \phi_{2}=0, \frac{\partial \phi}{\partial n}=\frac{\partial \phi_{1}}{\partial n}+\frac{\partial \phi_{2}}{\partial n}=\frac{\partial w}{\partial t}, P \in S_{1} .
$$

$$
\frac{\partial \phi}{\partial n}=\dot{\zeta}, P \in S_{0} ; \dot{\phi}+g \zeta+a_{s}(t) x=0, P \in S_{0}
$$


From (9) and (12) we have

$$
\frac{\partial \phi_{2}(x, y, z, t)}{\partial n}=\sum_{k=1}^{n} \dot{d}_{k}(t) \frac{\partial \phi_{2 k}(x, y, z)}{\partial n}=\dot{\zeta}
$$

So

$$
\frac{\partial \phi_{2}(x, y, z, t)}{\partial n}=\sum_{k=1}^{n} d_{k}(t) \frac{\partial \phi_{2 k}(x, y, z)}{\partial n}=\varsigma .
$$

We have $\dot{\phi}_{1}=0$ from relation (8). Then equality $\dot{\phi}+g \zeta+a_{S}(t) x=0$ leads to the system of differential equations

$$
\sum_{k=1}^{n} \ddot{d}_{k}(t) \phi_{2 k}(x, y, z)+g \sum_{k=1}^{m} \dot{c}_{k}(t) \frac{\partial \phi_{1 k}(x, y, z)}{\partial n}+g \sum_{k=1}^{n} d_{k}(t) \frac{\partial \phi_{2 k}(x, y, z)}{\partial n}=0 .
$$

Using for functions $\phi_{2 k}$ the relations $\frac{\partial \phi_{2 k}}{\partial n}=\frac{\kappa_{k}^{2}}{g} \phi_{2 k}, P \in S_{0} ; k=1, \ldots n$, we obtain

$$
\sum_{k=1}^{n}\left[\ddot{d}_{k}(t)+\kappa_{k}^{2} d_{k}(t)\right] \phi_{2 k}(x, y, z)+g \sum_{k=1}^{m} \dot{c}_{k}(t) \frac{\partial \phi_{1 k}(x, y, z)}{\partial n}=0 .
$$

Due to orthogonality of natural modes of fluid vibrations in rigid vessel we have after dot product of (14) by functions $\phi_{21}$

$$
\ddot{d}_{l}(t)+\kappa_{l}^{2} d_{l}(t)+\frac{g}{\left(\phi_{2 l}, \phi_{2 l}\right)} \sum_{k=1}^{m} \dot{c}_{k}(t)\left(\frac{\partial \phi_{1 k}}{\partial n}, \phi_{2 l}\right)+a_{s}(t)\left(x, \phi_{2 l}\right)=0, l=1,2 . ., n .
$$

When functions $\phi_{1 \mathrm{k}}$ and $\phi_{2 \mathrm{k}}$ are obtained, we substitute them in equation (2) and obtain the following equation:

$$
L\left(\sum_{k=1}^{m} c_{k} u_{k}\right)+M\left(\sum_{k=1}^{m} \ddot{c}_{k} u_{k}\right)=-\rho_{l}\left(\sum_{k=1}^{m} \ddot{c}_{k} \phi_{1 k}+\sum_{i=1}^{n} d_{i} \phi_{2 i}+g z+a_{s}(t) x\right)+Q .
$$

Let $\omega_{\mathrm{k}}, \mathrm{u}_{\mathrm{k}}$ be natural frequencies and free vibrations modes of the shell in vacuum. Then the following relationships are valid

$$
\boldsymbol{L} u_{k}=\omega_{k}^{2} \boldsymbol{M} u_{k},\left(\boldsymbol{M} u_{k}, u_{j}\right)=\delta_{k j} .
$$

Considering the result of dot product of equation (13) by $\mathrm{u}_{\mathrm{j}}$ and taking into account equations (14), we come to the next set of $n+m$ second order differential equations:

$$
\begin{gathered}
\ddot{c}_{j}(t)+\omega_{j}^{2} c_{j}(t)+\rho_{l} \sum_{k=1}^{m} \ddot{c}_{k}\left(\phi_{1 k}, u_{j}\right)+ \\
+\rho_{l}\left[\sum_{i=1}^{n} \dot{d}_{i}\left(\phi_{2 i}, u_{j}\right)+g\left(z, u_{j}\right)+a_{s}(t)\left(\rho, u_{j}\right)\right]=\left(Q, u_{j}\right), j=1, m \\
\ddot{d}_{l}(t)+\kappa_{l}^{2} d_{l}(t)+\frac{g}{\left(\phi_{2 l}, \phi_{2 l}\right)} \sum_{k=1}^{m} \dot{c}_{k}(t)\left(\frac{\partial \phi_{1 k}}{\partial n}, \phi_{2 l}\right)+ \\
+a_{s}(t)\left(x, \phi_{2 l}\right)=0, \quad l=1,2 . ., n .
\end{gathered}
$$


So, the scheme of the solution for considered problem consists of following steps. First, we have to obtain the natural frequencies and mode shapes of the free vibrations of elastic shell in vacuum. The problem is solved using FEM. Second, it is necessary to obtain the frequencies and free vibration modes of liquid in rigid shell under force of gravity. Then we define the frequencies and free vibration modes of elastic shell without including the force of gravity. These two problems are solved using BEM. And at the end we solve the set of second order differential equations using 4th and 5th order Runge-Kutta method.

We use furthermore the cylindrical coordinate system and represent unknown functions as Fourier series by circumferential coordinate

$$
w=w(r, z) \cos \alpha \theta, \phi=\phi(r, z) \cos \alpha \theta .
$$

To solve the coupled hydro-elasticity problem it is necessary to determine the potentials $\phi_{1}$ and $\phi_{2}$. These problems were reduced to the solution of the systems of singular integral equations. Determination of the potential $\phi_{1}$ was accomplished as in $[6,7]$.

To determine the potential $\phi_{2}$ we have to obtain functions $\psi_{\mathrm{k}}$. Let us denote by $\psi_{1 \mathrm{k}}$ the values of $\psi_{\mathrm{k}}$ on the wetted surface $\mathrm{S}_{1}$ and by $\psi_{0 \mathrm{k}}$ the values of $\psi_{\mathrm{k}}$ on the free surface $\mathrm{S}_{0}$. Using the BEM direct formulation and skipping for convenience an index $\mathrm{k}$ we can write the following system of singular integral equations

$$
\begin{gathered}
2 \pi \psi_{1}+\iint_{S_{1}} \psi_{1} \frac{\partial}{\partial n}\left(\frac{1}{r}\right) d S_{1}-\frac{\kappa^{2}}{g} \iint_{S_{0}} \psi_{0} \frac{1}{r} d S_{0}+\iint_{S_{0}} \psi_{0} \frac{\partial}{\partial z}\left(\frac{1}{r}\right) d S_{0}=0, \\
-\iint_{S_{1}} \psi_{1} \frac{\partial}{\partial n}\left(\frac{1}{r}\right) d S_{1}-2 \pi \psi_{0}+\frac{\kappa^{2}}{g} \iint_{S_{0}} \psi_{0} \frac{1}{r} d S_{0}=0 .
\end{gathered}
$$

Suppose that

$$
\psi=\psi(r, z) \cos \alpha \theta .
$$

We obtain for each harmonic the following system of singular integral equations

$$
\begin{gathered}
\iint_{S_{1}} \psi \frac{\partial}{\partial n}\left(\frac{1}{r\left(P, P_{0}\right)}\right) d S_{1}=\int_{r} \psi(z) \Theta\left(z, z_{0}\right) r(z) d \Gamma, \\
\iint_{S_{0}} \psi\left(\frac{1}{r\left(P, P_{0}\right)}\right) d S_{0}=\int_{0}^{R} \psi(\rho) \Phi\left(P, P_{0}\right) \rho d \rho .
\end{gathered}
$$

Here kernels $\Theta\left(z, z_{0}\right)$ and $\Phi\left(P, P_{0}\right)$ are defined as

$$
\begin{gathered}
\Theta\left(z, z_{0}\right)=\frac{4}{\sqrt{a+b}}\left\{\frac{1}{2 r}\left[\frac{r^{2}-r_{0}^{2}+\left(z_{0}-z\right)^{2}}{a-b} \mathrm{E}_{\alpha}(k)-\mathrm{F}_{\alpha}(k)\right] n_{r}+\frac{z_{0}-z}{a-b} \mathrm{E}_{\alpha}(k) n_{z}\right\} \\
\Phi\left(P, P_{0}\right)=\frac{4}{\sqrt{a+b}} \mathrm{~F}_{\alpha}(k) .
\end{gathered}
$$

For numerical solution of singular integral equation systems the boundary element method with constant approximation of unknown density on elements was used [5]. Integration by the fluid volume is reduced to integrals along the 
shell meridian and along the radius of the liquid free surface. It is the basic advantage of our method based on the combination of boundary integral equations method, FEM, BEM and expansion into Fourier series. It would be noted that the only FEM analysis requires 3D modeling to solve this coupled problem. That leads to essentially more computer timetable and it does not allow using effectively such methods in computer monitoring problems.

\section{Some numerical results}

We consider a semi-spherical shell filled with a fluid and having the following parameters: the shell radius is $R=5.08 \mathrm{~m}$, the thickness $h=0.0254 \mathrm{~m}$, the modulus of elasticity $E=70 \mathrm{GPa}$, Poisson's ratio $v=0.3$, the material's density is $\rho=2770 \mathrm{~kg} / \mathrm{m}^{3}$. The liquid is incompressible and its density is $\rho=1000 \mathrm{~kg} / \mathrm{m}^{3}$. The shell is assumed to be pin-connected over its contour (Figure 2a)).

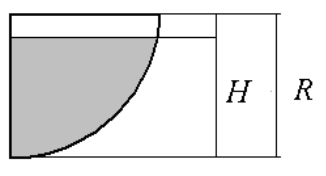

a)

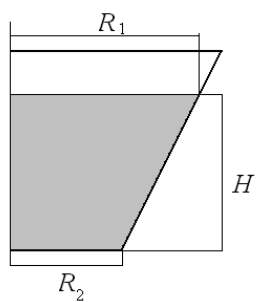

b)

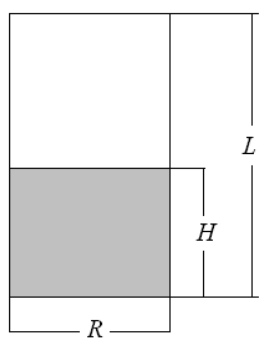

c)

Figure 2: Drafts of shells partially filled with the liquid.

The calculations of the natural vibrations of the sphere in vacuum and in liquid were carried out. Table 1 provides the numerical values of the natural frequencies of vibration for various numbers of nodal diameters $\alpha$.

Table 1: Comparison of frequencies for hemisphere with liquid.

\begin{tabular}{|c|c|c|c|c|}
\hline \multirow{2}{*}{$\alpha$} & \multirow{2}{*}{$\mathrm{m}$} & \multicolumn{3}{|c|}{ Natural frequencies, $\mathrm{Hz}$} \\
\cline { 3 - 5 } & & \multirow{2}{*}{ In vacuum } & \multicolumn{2}{|c|}{ With fluid } \\
\cline { 3 - 5 } & & & $\mathrm{BEM}$ & {$[10]$} \\
\hline \multirow{3}{*}{0} & 1 & 117.19 & 22.31 & 22.00 \\
\cline { 2 - 5 } & 2 & 146.17 & 33.63 & 33.38 \\
\cline { 2 - 5 } & 3 & 152.66 & 41.66 & 42.02 \\
\hline \multirow{3}{*}{1} & 1 & 87.938 & 22.28 & 22.44 \\
\cline { 2 - 5 } & 2 & 138.91 & 34.89 & 36.88 \\
\cline { 2 - 5 } & 3 & 150.34 & 43.21 & 48.92 \\
\hline \multirow{3}{*}{2} & 1 & 141.54 & 31.56 & 31.57 \\
\cline { 2 - 5 } & 2 & 151.37 & 40.61 & 41.55 \\
\cline { 2 - 5 } & 3 & 154.94 & 47.56 & 50.29 \\
\hline
\end{tabular}


The obtained numerical results are compared with those reported by Mokeev [10] for a compressible fluid.

The mode shapes of the shell meridian for $\alpha=0$ and various $m$ are shown in Figure 3. Here the solid line denotes the shell meridian, a long dotted line denotes the mode shapes in vacuum, and a short dotted line denotes the mode shapes in the fluid.
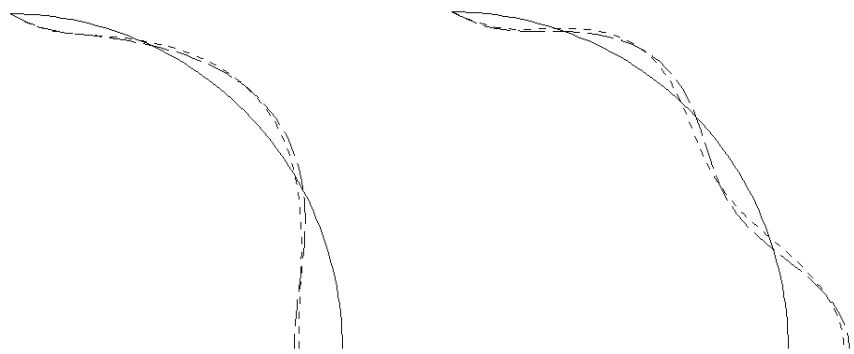

Figure 3: The mode shapes of vibration in vacuum and with fluid: $\alpha=0$, $\mathrm{m}=2,3$.

The problems on liquid free vibrations in rigid shells including gravity were under consideration. As an example we put the numerical results for the conical shell (Figure 2b)). The analytical values of free vibration frequencies are defined by following formulas [11]

$$
\kappa^{2}=\frac{g}{R} \lambda_{0} \operatorname{th}\left(\lambda_{0} \bar{H}\right), \quad \psi(\bar{x}, \bar{y}, \bar{z})=\frac{\operatorname{ch}\left(\lambda_{0} \bar{z}\right)}{\operatorname{ch}\left(\lambda_{0} \bar{H}\right)} \bar{\psi}(\bar{x}, \bar{y}),
$$

where $\bar{x}=x / R_{1}, \bar{y}=y / R_{1}, \bar{z}=z / R_{1}, \bar{H}=H / R_{1} ; R_{l}$ is the radius of free surface, $R_{2}=0 ; \mathrm{H}$ is the filling level in the tank, $\lambda_{0}$ are roots of equation $I_{\alpha}^{\prime}(\lambda)=0 ; I_{\alpha}(r)$ are Bessel functions of the first kind.

At numerical simulation we use the net of $n$ boundary elements on the shell meridian and $\mathrm{m}$ boundary elements on the free fluid surface. Table 2 represents the numerical values of two first own values $\kappa^{2} R / g$ for $\alpha=0$ and $\alpha=1$ at different $\mathrm{n}$ and $\mathrm{m}$. Here are also the analytical values obtained by formula (19).

Table 2: $\quad$ Convergence of own values in dependence of the net.

\begin{tabular}{|c|c|c|c|c|}
\hline \multirow{2}{*}{$n+m$} & \multicolumn{2}{|c|}{$\alpha=0$} & \multicolumn{2}{c|}{$\alpha=1$} \\
\cline { 2 - 5 } & 1 & 2 & 1 & 2 \\
\hline $10+10$ & 3.54 & 6.93 & 1.39 & 5.15 \\
\hline $20+20$ & 3.50 & 6.78 & 1.38 & 5.05 \\
\hline $30+30$ & 3.48 & 6.74 & 1.37 & 5.02 \\
\hline $40+40$ & 3.47 & 6.71 & 1.37 & 5.01 \\
\hline Solution $[12]$ & 3.46 & 6.70 & 1.36 & 4.97 \\
\hline
\end{tabular}


Figure 4 demonstrates the own modes corresponded to the two lowest frequency parameters of liquid vibrations on free surface. The numerical data testify that proper accuracy of calculating a frequency $(1.6 \%)$ is achieved at the net of $20+20$ boundary elements.
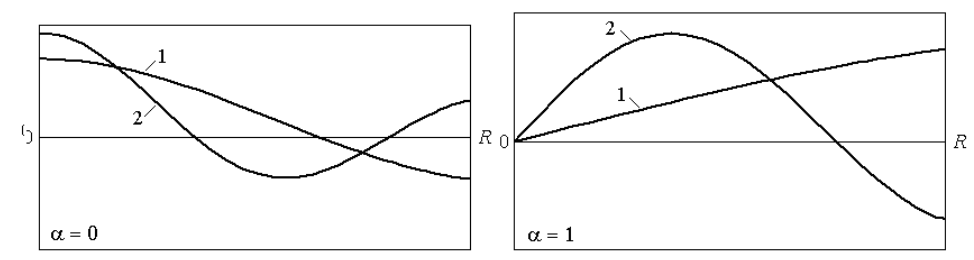

Figure 4: First and second vibration modes of conical shell at $\alpha=0$ and $\alpha=1$.

The forced vibration problem for above-mentioned hemisphere (Figure 2a)) was investigated. The impulse load $\mathrm{Q}(\mathrm{r}, \vartheta, \mathrm{z}, \mathrm{t})=\mathrm{P}(\mathrm{r}, \vartheta, \mathrm{z}) \Theta(\mathrm{t}), \mathrm{P}=$ const was considered in the following form

$$
\Theta(t)= \begin{cases}1, & t \leq T_{1} \\ 0, & t>T_{1}\end{cases}
$$

where $\mathrm{T}_{1}=0.002 \mathrm{~s}$. Figure 5 shows the time history of axial displacement. In papers [6-7] the coincidence of results obtained by proposed method and program complex ANSYS was demonstrated.
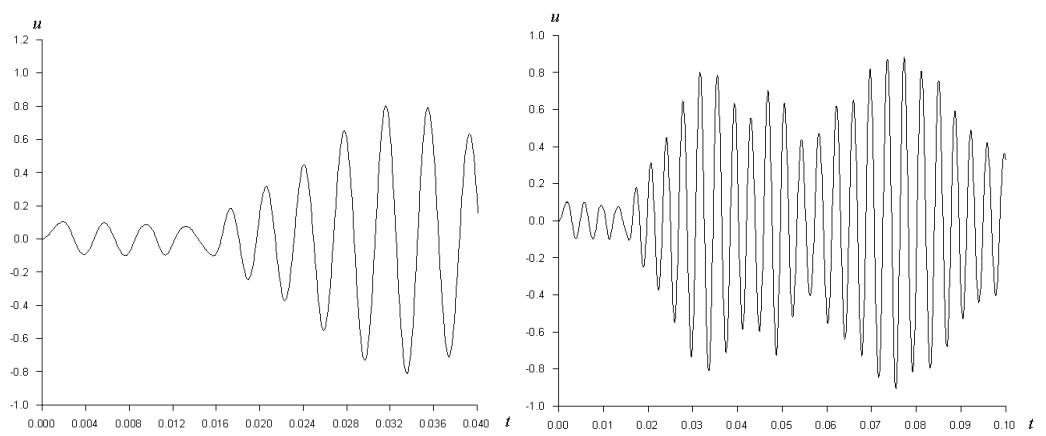

Figure 5: Time history of axial displacement.

The shell scheme and time history of radial displacement at different points are shown at Figure 6.

The rigid cylindrical shell (Figure 3c)) was considered under seismic loading. Figure 7 shows the accelerogram of El-Centro earthquake [12] in USA, 1940 (magnitude 9 on the Richter scale) and the position of free surface at the initial time. The parameters of shell were following: the radius $\mathrm{R}=1 \mathrm{~m}$, the thickness $\mathrm{h}=0.01 \mathrm{~m}$, the length $\mathrm{L}=2 \mathrm{~m}$, Young's modulus $\mathrm{E}=2 \cdot 10^{5} \mathrm{MPa}$, Poisson's ratio $\nu=0.3$, the material's density $\rho=7800 \mathrm{~kg} / \mathrm{m}^{3}$, the fluid density $\rho_{\mathrm{l}}=1000 \mathrm{~kg} / \mathrm{m}^{3}$. The filling level of the fluid is denoted as $\mathrm{H}$. Boundary conditions are following: $\mathrm{ur}=\mathrm{uz}=\mathrm{u} \theta=0$ to $\mathrm{z}=0$ and $\mathrm{r}=\mathrm{R}$. Here we assume $\mathrm{H}=\mathrm{L}$. 

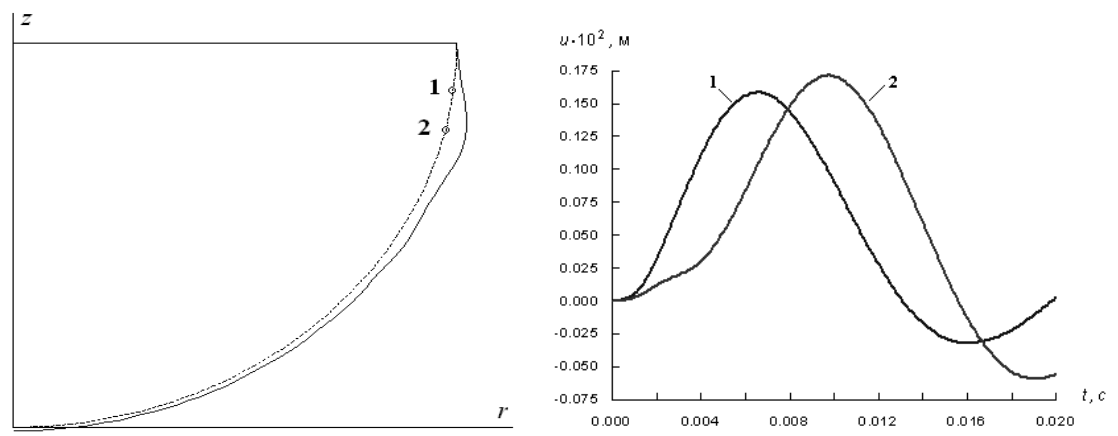

Figure 6: Shell scheme and time-history of radial displacement.
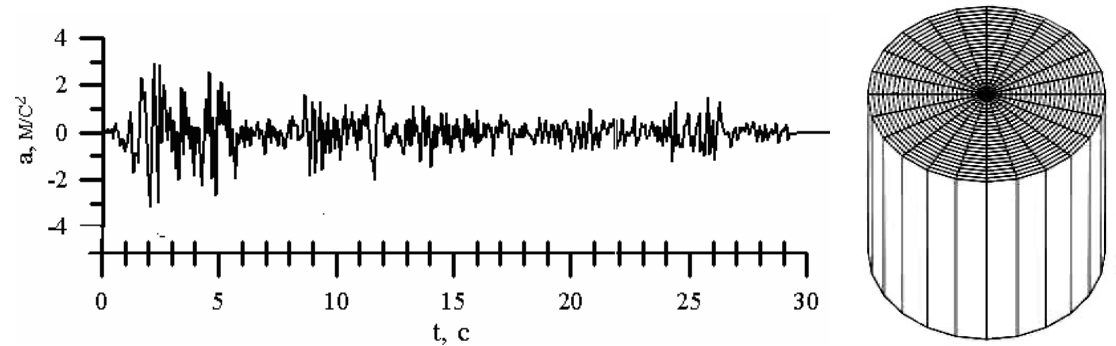

Figure 7: Accelerogram of El-Centro earthquake and free surface at the initial time.

Figure 8 demonstrates free surface of liquid in cylindrical tank at different times that was calculated using system (18) for the accelerogram $a_{s}(t)$ presented in Figure 7.
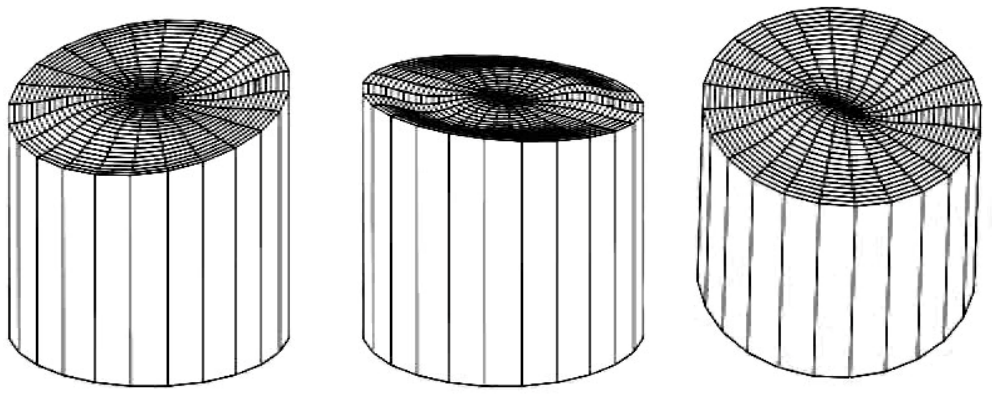

Figure 8: $\quad$ Free surface of liquid at different times. 


\section{Conclusions}

The numerical procedure based on a coupling finite and boundary element methods and expansion into Fourier series is developed for free and forced vibration analysis including seismic loading for shells of revolution with an arbitrary meridian. The shells considered were partially filled with the inviscid incompressible fluid. We introduce the representation of the velocity potential as the sum of two potentials, corresponding to the problems of fluid and shell free vibrations. The fluid vibrations were considered in the rigid shell and the shell vibrations were studied for weightless contained fluid. Both problems were solved using reduced BEM. Integration by the fluid volume was reduced to integrals along the shell meridian and along the radius of the liquid free surface. It is the basic advantage of our method. The governing integral equations for each harmonic have been obtained. The numerical simulation of free and forced vibrations for elastic and rigid shells was accomplished.

\section{Acknowledgements}

The authors gratefully acknowledge our collaborator on STCU Projects \#4624 and \# 5742 Professor Carlos Brebbia for his constant support and interest in our research.

\section{References}

[1] Coalland - Faces of Donetsk. Zoп Environment Network and UNEP/GRID-Arendal, Global Publishing Services, France, 2011, 44p., http://enrin.grida.no/donetsk

[2] Dukowicz, J. K., Dvinsky, and A. S., Approximate Factorization as a High Order Splitting for the Implicit Incompressible Flow Equations, J. Comput. Phys., 102, pp. 330-336, 1992.

[3] Tezduyar T. E. Finite Element Methods for Fluid Dynamics with Moving Boundaries and Interfaces. Encyclopedia of Computational Mechanics, (3): Fluids, pp. 1-55, 2004.

[4] Recent Advances in Boundary Element Methods A Volume to Honor Professor Dimitri Beskos Manolis, George; Polyzos, Demosthenes Eds., XXXVIII, 470 p., 2009.

[5] Brebbia, C.A., Telles, J.C.F. and Wrobel, L.C. Boundary Element Techniques, Springer-Verlag: Berlin and New York, 1984.

[6] Strelnikova E., Yeseleva E., Gnitko V., Naumenko V. Free and forced vibrations of the shells of revolution interacting with the liquid Proc. of XXXII Conference "Boundary elements and other mesh reduction methods" WITPress, Transaction on Modeling and Simulation, P. 203-211, 2010.

[7] Ventsel E.,. Naumenko V, Strelnikova E., Yeseleva E. Free vibrations of shells of revolution filled with a fluid. Engineering analysis with boundary elements, 34, pp. 856-862, 2010. 
[8] Gnitko V., Marchenko U., Naumenko V., Strelnikova E. Forced vibrations of tanks partially filled with the liquid under seismic load. Proc. of XXXIII Conference "Boundary elements and other mesh reduction methods" WIT Press, Transaction on Modeling and Simulation, P. 285-296, 2011.

[9] Ibrahim R. A. Liquid sloshing dynamics: theory and applications Cambridge University Press, 957p., 2005.

[10] Mokeev V.V. On a solution of matrix equations in the Lagrange finite element modeling of fluid in the problems of the hydroelasticity, Dynamics and Strength of Machines pp. 11-17, 1999 (in Russian).

[11] Lukovsky I. A. Introduction into non-linear dynamics of rigid bodies with fluid filled cavities. - Kiev: Naukova dumka,-296 pp, 1990 (in Russian).

[12] Paz M. International handbook of earthquake engineering: codes, programs, and examples: Oxfordshire, UK: Chapman and Hall, 545 p. 1994. 